\title{
HELLP Syndrome: a 5 years retrospective review
}

R. Torgal, R. Cerqueira Vieira, A. Silva, M. Rocha, I. Guedes, J. P. Assunção Anaesthesiology Department, Centro Hospitalar Tondela-Viseu

\section{Background and Goal of Study}

HELLP Syndrome is characterized by hemolysis, elevated liver enzymes and low platelet levels. It's an important obstetric complication which is, either a variant of pre-eclampsia, or an entity of its own. It usually presents with elevated blood pressure and a variety of symptoms.

We reviewed the anesthetic management of women diagnosed with HELLP syndrome in our institution in 5 years and describe the outcomes.

\section{Materials and Methods}

- Retrospective, observational study from January 2012 to December 2016.

- Included all women with diagnosis of HELLP syndrome.

-Data were collected from physical and electronic records.
- Variables included:

- Age, obstetric history, type of delivery, type of anesthesia, maternal and fetal outcome and complications, ICU admission and discharge date.

- All data was compiled and analyzed using SPSS version 22

\section{Results and Discussion}

antihypertensive drugs, as well as seizure prophylaxis

- There were 12 pregnant women diagnosed with with magnesium sulfate.

HELLP syndrome in the 5 years period, with an - Emergency cesarean section was performed in 10 incidence of 1:809 deliveries $(0,12 \%)$. women, using general anesthesia in 6 of them.

- The syndrome was classified antepartum in $7 \cdot \mathrm{ICU}$ admission was necessary in 4 cases. patients. The median age was 33 years and $66,6 \%$. The median time until discharge was 9 days. presented after the 35th week.

- In 7 cases the syndrome developed in the first pregnancy.

- There were 2 fetal deaths in patients arriving to the hospital with no fetal heart rate in cardiotocography and 1 baby was admitted to NICU after cesarean - All women received corticosteroid treatment and delivery.

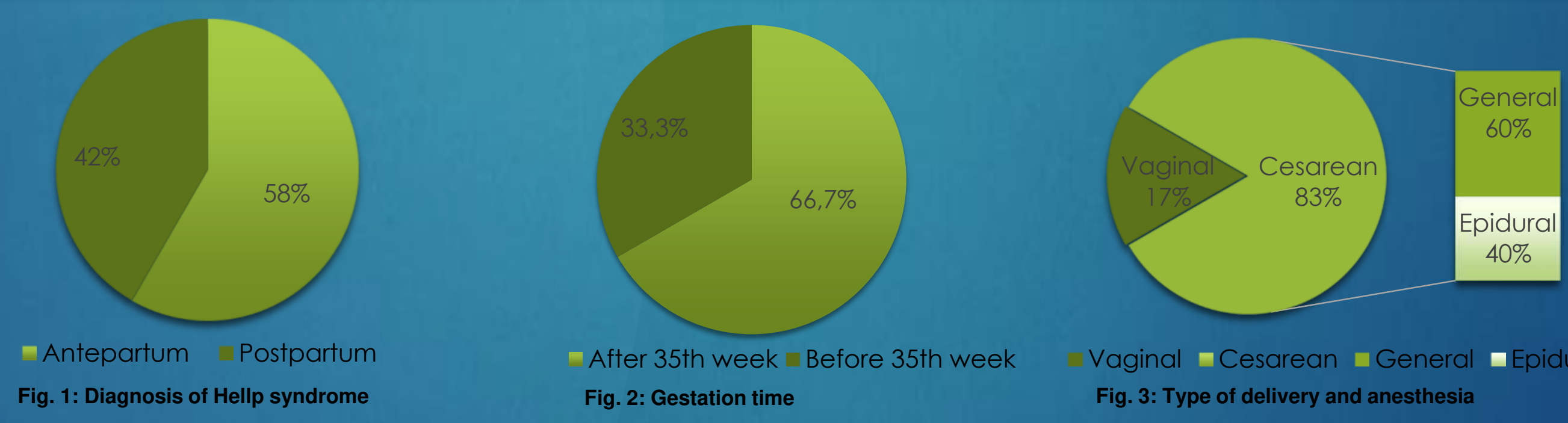

\section{Conclusions}

HELLP syndrome is an obstetric emergency in which a multidisciplinary approach (obstetricians, pediatricians and anesthesiologists) is vital to a successful outcome. Early recognition of signs and symptoms and prompt treatment is necessary to decrease maternal and fetal morbimortality. 